\title{
QUELQUES ASPECTS DE LA REPRODUCTION CHEZ LES POISSONS
}

\author{
par Y. FRANÇOIS \\ Assistant a la Station Centrale'd'Hydrobiologic appligude.
}

\section{Les Caractères sexuels secondaires.}

Chez les animaux à sexes séparés (et les Poişsons sont du nombre dans leur quasi totalité) la différence essentielle entre les sexes réside essentielkement dans la nature des glandes génitales (ovaires ou testicules) et lit structure du tractus génital, c'est-à-dire des.organes et conduites annexés à ces glandes (oviducte ou canal déférent, vésicules séminales, etc...). L'ensemble constitue les caractères sexuels primaires.

Mais les sexes peuvent se distinguer extérieurement par des différences plus ou moins accentuées du reste du corps ou par un comportement différent, Ce sont les caractères sexuels secondaires.

Aingi, pour prendre un exemple familier, c'est par son plumage, par la crete et les ergots, mais aussĩ par sa combativité et son chant, que le cơ se distingue de la poule.

Chez les Cervidés, les cornes du mâle sont bien plus développées que chez la femelle.

Dans le monde des Poissons, les différences sont généralement moins marquées et portent principalement sur la coloration. Le male, à la période de reproduction, acquiert des couleurs très vives, à tons rouges et jaunes, dues au développement, dans la peau, de grosses cellułes pigmentaires. A cette pigmentation très riche, on donne le nom de " parure de noces " ou de a livrée nuptiale ".

On l'observe, par exemple, chez le Vairon, et aussi chez la Bouvière. Dans cette espèce, en outre, la femelle développe, au, moment de la ponte, un long tube ovipositeur qui prolonge extérieurement l'oviducte.

Quelques Poissons exotiques, dont l'élevage en aquarium 'est' aujourd'hui très répandu, montrent des caractéres sexuels secondaires encore plus marqués et permanents. Citons le Xiphơphore dont le mâle porte une longue épée postérieure développée à partir des rayons de la nageoire caudale.

Aucune indication aussi marquée dans la forme ou dans la coloration, chez les Poissons cultivés, ne sépare les individus des deúx sexes. Toute- 
fois, les éleveurs savent bien, au moins au moment de la reproduction, distinguer les males et les femelles.

Pour la Truite et les Salmonidés, cette distinction est-difficile en temps normal. On indique généralement que les males ont la tête plus petite et la mâchoire inférieure plus forte que les femelles. Mais ces caractères ne sont guère marqués qu'après la première fraye.

Et, a ce moment, il est apparu un caractère bien plus net : la femelle présente, contre la nageoire anale, une pupille urogénitale saillante ou débouxchent, en arrière de l'anus, lé pore génital et le pore urinaire. Cette papille est rudimentaire chez le mâle.

Chez la Tanche, le dimorphisme sexuel est encore plus accusé et, surtout, il est constant. Les nagéoires ventrales ont des rayons semblables en épaisseur, chez les femelles; chez les males au contraire, le deuxième rayon extérieur est plus épais et plus robuste que les autres ; ce, caractere est très apparent.

En outre, pendant la période des amours; la pigmentation devient plus brillante chez le malle, plus sombre chez la femelle dont les flancs sont élargis, gonflés par la masse des milliers d'oufs mars.

\section{Les périodes de reproduction.}

La plupart des Poiseons de nos pays pondent seulement une fois par an. L'époque de la reproduction est asseż, strictement déterminée pour une espèce donnée et ne subit que des variations d'assez faible amplitude: Mais elle varie largement d'une espèce à l'autre.

En ce qui concerne seulement les Poiseons d'eau donce de France, on peut dire qu'aucun ne se reproduit entre Juillet et Novembre. La périodę de reproduction des diverses espèces s'échelonne, de façon à peu près continue, de Novembre à Juillet.

Suivant l'époque de la fraye, on répartit nos Poissons en trois groupes.

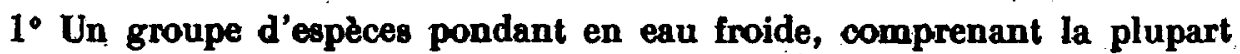
des Salmonidés et la Lote (Gadidés). C'est ce que LGGEn appelle le groupe d'hiver.

Voici les époques de ponte de quelques espèces de ce groupe :

Salmonidés :

Salmo fario........ 15 Novembre-fin Janvier.

S. irideus......... fin Décembre-début Février.

S. fontinalis........ fin Novembre-Décembre.

Corégones ......... $1^{\text {or }}$ Décembre-1 ${ }^{\text {or }}$ Mars.

Gadides :

Lota lota......... Décembre-Janvier. 
2* Groupe de printemps, pondeur en eau tempérée comprenant les Poiscons carnasgiens et 2 Salmonidés tardifs.

Brechet $\ldots \ldots \ldots \ldots \ldots$
Perche $\ldots \ldots \ldots \ldots \ldots$
Chabot $\ldots \ldots \ldots \ldots \ldots$

$3^{\circ}$ Groupe d'eté, comprenant les pondeurs d'eau chaude c'est-à-dire les Cyprinidés et quelques autres espèces:

Carpe $\ldots \ldots \ldots \ldots \ldots \ldots$
Tanche $\ldots \ldots \ldots \ldots \ldots$
Gardon $\ldots \ldots \ldots \ldots \ldots$
Black Bass $\ldots \ldots \ldots \ldots$
$\begin{aligned} & \text { Idem.-Juillet. } \\ & \text { Idem. }\end{aligned}$

Les gametes et la fécondation.

Chez les Poissons, comme chez tous les animaux supérieurs, chaque individu a pour origine ane celle cellule; l'œuf, provenant de la fusion de deux cellules anormales, fortement différenciées, noyau incomplet, les éléments' sexuels ou gamètes : l'ov̀ule ou gaméte femelle est une cellule volumineuse, immabile, chargée de substance de réserve; il est produit par l'ovaire. Le spermatowolde ou gamete molo, produit dans lé testicule; eat au contraire une collule minusenle, trto motolle, eo contenant pas de réperve, preque réduite $A$ son noyau.

La focondation, cesta-ding la penétrition d'tn spermatosoide dans un ovule, est suivie de la fusion des noyaux des gamètes pour constituer le noyau complet; normal, de l'ceuf.

En outre, la fécondation déclenche la division de l'beuf ; c'est l'activation.

Les dciux gametes ne peuvent avoir une vie autoname et sans la fécondation, ils meurent et degénèrent rapidement.

Chez la wlupart des Poissons, lá fécondation eat externe et s'opère dans l'eau. Géntralement, ta femelle dépose ses ceuf séparément ou en amas, sur le fond on sur des plantes aquatiques, moing que tres legers ils ne Nottent enitre deux exux. Le mAle passe ensurite répand son sperme sur les ceufs frafchement pondus.

Struoture des ceufs de Salmonidés. - Les Salmonidés pondent des ceufs particulièrement volumineux. Celui de la Truite, par exemple, mesure 4 a 5. millimètres de diamètre. Sa masse principale est constituée par des substancés de réserve complexes, enclaves de la mätière vivante, qui constituent le vitellus.

Le protoplasme vivant est réduit à une petite lame discoìdale, à la surface du vitullus, le disque germinatif, qui représente le germe du futur embryon. 
Enfin, une coque élastique et résistante, assez épaisse, la membrane vitelline entoure l'wuf complètement. Elle présente à sa surface, au voisinage du disque germinatif, une dépression aú fond de laquelle s'ouvre un petit canal, le micropyle, par où, lors de la récondation, pénètrera le spermatozoide.

Tandis qu'il est mou dans la cavité abdominale de la femelle, l'ovule devient, après un court séjour dans l'eau, dur et élastique. Cette modification de sa consistance est due à l'absorption d'eau qui tend fortement la membrane et rend l'cuf turgescent. La membrane elle-même se gonfle par imbibition, de telle sorte que bientôt le micropyle se rétrécit et finit par s'oblitérer. Dès lors - et nous y reviendrons - la fécondation est impossible.

La structure des coufs des autres Poissons ne differe pas essentiellement dé celle des cufs de Truite. Les principales variations portent sur la grosseur de l'ceuf (c'est-d̂-dire en définitive sur la quantité de vitellus) et sur la nature de la membrane qui l'entoure et qui, pariois plus ou moins gluante, permet la fixation des cufs sur un support quelconque.

\section{Le spermatozoide et sa physiologie.}

Le spermatozoride est, nous l'avons dit, une cellule minuscule, schématiquement constituée par une tête ovolde munie d'un long fouet vibiratile qui est un organe locomateur. La longueur d'un spermatozoide de Truite est de l'ordie de $50_{\mu}(=1 / 20$ de millimetre) flagelle compris.

Les spermatozotdes sont émis par millions, enrobes dans un liquide plus ou moins epais, le sperme ou laitance.-Dang' fe sperme, au moment ou ils sont expulsés, les spermatozö̀des sont immobiles. C'est seulement au contact de l'eau qwo le flagelle commence à battre et que le spermatoroide se met en mouvement.

Mais, des observationg anciennes d'un biologiste franciais (HENnecur, 1877) ont montré que, dans l'eau, les spermatozoldes n'ont qu'une existence très éphémère.

D'après Hennegur, après 15 secondes de sejour dans l'eau, les spermatozoldes de Truite n'ont plus que des mourements lents et peu étendus. Beaucoup sont déjà immobiles. Néanmoins 60 œufs fécondés avec ce sperme ont encore donné 46 éclosins.

Plus récemment; des auteurs allemands ont repris les expériences de Hennegux, en les précisant et les complétant (Scheuruic et Gaschotr, Hakmiper, 1934). Hakmpel, par exemple, a mesuré avec précision la duré de la mobilité des spermatozoldes de différentes especes, a partir du moment où ils sont mis au contact de l'eau.

Une goutte de sperme est déposée sur une lame porte-objet et on y ajoute unc goutte d'eau. On observe au microscope les mouvements des sperma- 
tozoides qui s'agitent en tous sens puis se calment très vite. On note le moment où la plupart d'entre eux demeurent immobiles. Des mouvements du fiagelle penvent persister encore quelques temps, mais sont insuffisants pour déplacer le petit organisme.

\begin{tabular}{|c|c|}
\hline Espèces & Durée des mouvements \\
\hline Salmo fario... & 23 secondes. \\
\hline s. irideus. & $40 "$ \\
\hline 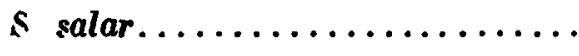 & 45 \\
\hline Barbus fluvialitis (Barbeau)...... & 2 minutes \\
\hline Esox lucius (Brochet) . ......... & 2 \\
\hline Cyprinus carpio (Carpe). & 5 \\
\hline
\end{tabular}

Ces chiffres montrent combien est brève la durée de vie des spermatozoïdes, particulièrement ceux des Salmonidés.

\section{La fécondation artificielle.}

La coñnaissance de ces faits relatifs à la biologie des spermatozoïdes explique clairement les excellents résultats obtenus par la méthode de la fécondation artifloielle; ils justifient sa pratique en salmoniculture.

Pour la Truite, dans les conditions naturelles on estime que 10 \& $15 \%$ des ceufs pondus seulement sont fécondés et se développent. La médiocrité de ce rendement est évidemment en rappart avec le fait que les spermatozoîdes n'ont que quelques secondes pour accomplir leur fonction. Tous ceux qui, au cours de ce bref laps de temps, ne rencontrent pas un œuf, sont perdus. Mais réciproquement les œufs non fécondés ne pourront se développer.

Et l'on se rappelle aussi que les œufs vierges ne sont fécondables que pendant la demi-heure qui suit leur ponte dans l'eau (HenNegur) car le gonflement de la membrane finit par fermer le micropyle.

Ainsi, lorsqu'ils sont émis dans l'eau, les gamètes femelles et surtout mâles perdent bientôt la possibilité de se fusionner.

Au contraire on a remarqué depuis longtemps que les oeufo vierges et la laitance sont susceptibles de se conserver plusieurs jours aveo toutes leurs propriétés, lorsqu'ils sont conservés d sec, dans une atmosphère froide et humide.

Henneguy, par exemple, a observé que de la laitance laissée pendant 4 jours dans un flacon bouché à une température de $10-15^{\circ} \mathrm{C}$. avait conservé à peu près tout son pouvoir fécondant; sur 40 ceufs fécondés avec lui, 8 seulement ne se sont pas développés.

Un refroidissement de courte durée, quelques degrés au-dessous de zéro, peut être supporté par les spermatozoĩdes, mais la congélation amène cependant des dommages assez sérieux. La meilleure température pour la 
conservation dı: sperme paraft être $3-5^{\circ}$ au dessus de 0 . Et il est bon, de ne pas depasser $15^{\circ}$.

Les aiufs perdent leur vitalité un peu plus vite, semble-t-il, mais possèdent encore, après 3 jours (â $12^{\circ}$ ), leur propriété d'être fécondés (HETNEGUY).

La fécondation artificielle (dont la technique, d'ailleurs très simple, est suffisamment connue pour qu'il soit nécessaire de la décrire) est aujourd'hui pratique absolument généralisée dans les établissements salmonicoles. Mais ce n'est pas une nouveauté, si l'on en croit un vieil ouvrage de pisciculture :

" Vers le milieu du siècle dernier, en 1758, le Comte de Golsterv, grand chancelier des duchés de Bergues et de Juliers pour son Altesse palatine, remit à l'un des ancêtres du célèbre Fourcroy, un mémoire sur la fécondation artificielle des ceufs de Poissons et sur l'emploi de ce procédé pour le repeuplement des rivières et des étangs. Ce remarquable travail dont JACOBI était l'auteur était écrit en allemand et le Comte de GoLstern voulut bien le lui donner en latin. La version française fut publiée, en 1773, dans le Tnaité général des Péches, de Dumamel De Moncead, rédigé par ordre de l'Académie des Sciences ". (Coste, Instructions pratiques sur la Pisciculture, Paris 1853).

Si la fécondation artificielle ne se pratique pas pour les Cyprinidés, il y a à cela quelques raisons. D'abord la physiologie des spermatozoîdes de Carpe montre qu'elle n'est pas nécessaire. Ceux-ci ont une durée de vie, dans l'eau, dix fois supérieure à celle des spermatozoìdes de Truite. Dans les cinq minutes que dure leur mobilité, ils ont le temps de remplir leur rôle et de féconder un œuf à côté duquel ils auront été déversés.

En outre, la Carpe pond ses ceufs l'un après l'autre et leur surface gluante les fixe aux plantes aquatiques. Si l'on provoque la ponte par pression sur le ventre d'une femelle mure, les œufs sont expulsés en amas, agglutinés et aỉnè difficilement técondables.

Toutefois, on a réussi la fécondation artificielle chez la Sandre (Sander lucioperca, Fam. des Percidés) dont les œufs, par le mucus qui les entoure sont comparables à ceux de la Carpe. Il est nécessaire, alors d'agiter vigqureusement les œufs recueillis de manière à éliminer le mucus gluant. Ainsi les œufs s'isolent et l'on peut les étaler sur des cadres où ils seront facilement fécondés. On l'a également pratiquée chez le Brochet et chez la petite Alose sédentaire des lacs italiens (Alosa lacustris). 